\title{
Acesso de mulheres lésbicas aos serviços de saúde à luz da literatura
}

\author{
Access to health services for lesbian women: a literature review
}

Adriane das Neves Silva (https://orcid.org/0000-0001-5383-2618) ${ }^{1}$

Romeu Gomes (https://orcid.org/0000-0003-3100-8091) ${ }^{1}$

${ }^{1}$ Instituto Fernandes Figueira, Fundação Oswaldo Cruz. Av. Rui Barbosa 716, Flamengo. 22250-020 Rio de Janeiro RJ Brasil.

adrianeves@gmail.com

\begin{abstract}
This study explores access to health services for lesbians in the light of current literature. A literature search was conducted using various databases and an interpretive synthesis of the findings of the selected articles was produced anchored in the concepts of habitus and symbolic violence developed by Pierre Bourdieu. Two main themes and their respective units of meaning were identified: (a) barriers and difficulties experienced by lesbians in accessing healthcare (issues related to coming out as a lesbian and difficulties experienced by health services and professionals in dealing with lesbian women); and (b) lesbian women's experiences in health services (unequal care, invisibility, and feeling uncomfortable). We conclude that, despite advances in policy and care protocols, sexual and gender diversity needs to be widely discussed in social, educational, and health settings.
\end{abstract}

Key words Female homosexuality, Lesbians, Healthcare access
Resumo O trabalho tem como objetivo explorar como se afiguram as especificidades do acesso de lésbicas aos serviços de saúde à luz da literatura. Como metodologia, realizou-se uma pesquisa bibliográfica e, em seguida, com base nos achados do acervo analisado, produziu-se uma sintese interpretativa ancorada em aspectos teóricos de Pierre Bourdieu. Em relação aos resultados, destacam-se duas temáticas com seus respectivos núcleos de sentidos: (a) barreiras e dificuldades do acesso de lésbicas à atenção à saúde (questões relacionadas à revelação de ser lésbica e dificuldades de os serviços e profissionais de saúde lidarem com essas mulheres) e (b) experiências das lésbicas nos serviços de saúde (atendimento desigual, invisibilidade $e$ constrangimento). No que se refere a conclusões, dentre outros aspectos, observa-se que, apesar dos avanços em relação a políticas e protocolos de atendimento a população em questão, as diversidades sexual e de gênero devem ser amplamente debatidas nos espaços sociais, de formação e de atenção à saúde.

Palavras-chave Homossexualidade feminina, Lésbicas, Acesso à atenção à saúde 


\section{Introdução}

Quando se olha para distintos segmentos populacionais, em alguns países, observa-se que o acesso aos serviços de saúde pode ser apresentar déficits ou não ter a qualidade que deveria ter. Esses problemas devem ser vistos dentro de um cenário que envolve aspectos políticos, econômicos, sociais, organizativos, técnicos e simbólicos ${ }^{1}$.

Colocando o foco nas mulheres lésbicas, afiguram-se especificidades de problemas que podem interferir no acesso aos serviços e na integralidade da atenção à saúde. Uma parte desses problemas fazem interseção com os das mulheres heterossexuais e bissexuais, uma vez que comumente ocorre uma negação da sexualidade feminina em geral $^{2}$. No campo de reivindicações políticas, o movimento de lésbicas nasce e, até certo ponto, se mantém na interseção do movimento homossexual em geral com o movimento feminista, ainda que busque autonomia e visibilidade.

No Brasil, apesar de assegurada pelo Sistema Único de Saúde uma assistência universal, integral e equânime, em 2006, Facchini e Barbosa ${ }^{3}$ observavam que a invisibilidade e a ausência de políticas adequadas afetavam o atendimento nos serviços de saúde e a atuação dos profissionais, que na ausência de informações sobre essa população, agem a partir de estereótipos socialmente disseminados.

Estudo de Valadão e Gomes ${ }^{4}$, com bases em artigos e em documentos publicados até 2008, encontrou problemas de acesso e de atenção à saúde dessas mulheres e, com base em Bourdieu, concluíram que as lésbicas eram invisíveis e, em alguns casos, eram vítimas de violência simbólica.

Alguns avanços, no campo das políticas de saúde, ocorreram após a publicação desse estudo, como é o caso - no Brasil - da promulgação da Política Nacional de Saúde Integral de Lésbicas, Gays, Bissexuais, Travestis e Transexuais. Resta saber se, com esses avanços, tanto no Brasil quanto em outros países, atualmente, ainda persistem problemas no acesso e na qualidade dos serviços relacionados às lésbicas ${ }^{5}$.

$\mathrm{Na}$ atualização da discussão do assunto em pauta, é importante não só sistematizar os resultados dos diferentes estudos publicados, mas também ensaiar uma interpretação que ajude na melhor compreensão acerca do conhecimento sobre o acesso de lésbicas aos serviços de saúde. Para isso, assim como Valadão e Gomes ${ }^{4}$, acredita-se que a perspectiva de Bourdieu pode ajudar nesse empreendimento. Dentre os aspectos dessa perspectiva utilizados por esses autores mencio- nados, entende-se que os conceitos de habitus e o de violência simbólica de Bourdieu ainda sejam úteis para melhor compreender o cenário atual.

O habitus, na teoria de Bourdieu ${ }^{6-9}$, se refere a um conhecimento adquirido, um haver, uma capital, indicando uma disposição incorporada. Corresponde a matrizes social e historicamente construídas, cujo exercício será determinado pela posição social do indivíduo, permitindo-lhe pensar, ver e agir nas mais variadas situações. Para o autor, diferentemente da palavra hábito, que se associa a algo cristalizado, a expressão habitus envolve uma capacidade criadora, ativa e inventiva. Dentro desse raciocínio, o sujeito receberia e reinventaria a "herança" para a formação do habitus $^{6-9}$.

Em relação ao conceito de violência simbólica, Bourdieu ${ }^{10}$ a situa na esfera da dominação, observando que há duas formas de se manter alguém sob o domínio: violência aberta e violência simbólica, sendo esta última entendida como aquela que é eufemizada, branda e invisível ${ }^{10}$. Para ele, de um lado, enfatizar a violência simbólica não significa minimizar o papel da violência física ou fazer esquecê-la e, por outro lado, é importante que não se entenda a violência simbólica como oposta à violência real, uma violência meramente espiritual, sem efeitos reais ${ }^{11}$. O autor considera ainda que a violência simbólica não é exercida a partir da lógica das consciências conhecedoras, mas sim através da lógica da obscuridade das disposições do habitus ${ }^{8}$.

Habitus e violência simbólica, na visão de Bourdieu, podem ser diretamente imbricados numa interpretação da literatura acerca do assunto aqui tratado, uma vez que em uma de suas obras ele discute, à luz desses conceitos, a heteronormatividade como oposição à homossexualidade ${ }^{11}$.

A partir dessas considerações iniciais, o estudo tem como objetivo explorar como se afiguram as especificidades do acesso de lésbicas aos serviços de saúde à luz da literatura.

\section{Método}

O desenho do estudo, inicialmente, se caracteriza como como uma pesquisa bibliográfica de abordagem qualitativa, tendo como fonte o artigo científico. Esse tipo de pesquisa, além de possibilitar o alcance de um amplo número de informações e dados dispersos em várias publicações, também auxilia na melhor definição do quadro conceitual que envolve o objeto ${ }^{12}$. 
O levantamento das publicações científicas foi realizado no período de novembro de 2018 a maio de 2019, nas seguintes bases de dados: Literatura Latino-americana e do Caribe em Ciências da Saúde (Lilacs), Base de dados bibliográficas especializada na área de Enfermagem (Bdenf), $U$. S. National Library of Medicine (PubMed), Medical Literature Analysis and Retrieval System Online (Medline) e Scientific Electronic Library Online (SciELO).

Nessas bases, ocorreu uma busca por meio de Descritores em Ciências da Saúde (DeCS), em português e inglês, combinados pelo operador booleano "and". Nas buscas pelas bases SciELO, Bdenf e Lilacs o descritor "homossexualidade feminina" foi combinado com "acesso aos serviços de saúde", "assistência integral a saúde", resultando em um número pequeno de artigos. Já na Medline e PubMed o número foi mais expressivo. Foram utilizados também os termos minorias sexuais e de gênero e homossexualidade, como tentativa de ampliar as buscas.

Nessa busca, foram estabelecidos os seguintes critérios de inclusão: somente foram selecionados artigos em função de sua grande circulação tanto no meio acadêmico como profissional, na íntegra em português, inglês e espanhol no período de 2004 a 2018, que descrevem a temática referente à saúde da mulher lésbica. Foi considerado o ano de 2004 como o início desse período, porque foi o ano da publicação da Política $\mathrm{Na}$ cional de Atenção à Saúde da Mulher ${ }^{13}$ que contempla as lésbicas nas ações de saúde da mulher. Como critérios de exclusão não foram incluídas as seguintes fontes: editoriais, cartas ao editor, dissertações e teses, artigos com enfoque exclusivamente clínico-epidemiológico e artigos, que não estavam disponíveis na íntegra.

O primeiro levantamento resultou em 273 artigos. Após a leitura dos títulos e/ou resumos foram eliminados 12 artigos duplicados e 192 pelos critérios de exclusão. Restaram 70 publicações que foram lidas na íntegra. Destas, 33 foram excluídos por não discutirem questões relacionadas ao acesso de lésbicas aos serviços de saúde, suas demandas e as especificidades da atenção dada a elas por esses serviços. Ao final, 36 artigos foram incluídos no estudo.

Os artigos foram analisados a partir de uma adaptação da técnica de análise de conteúdo, modalidade temática, descrita por Bardin ${ }^{14}$. Para essa autora, o tema é uma unidade de significação que se liberta do texto analisado e pode ser traduzi- do por um resumo, por uma frase ou por uma palavra. Com essa técnica, é possível identificar o que está por trás dos conteúdos manifestos ${ }^{15}$. Neste estudo, o tema está sendo entendido como uma categoria mais ampla que pode abranger mais de um núcleo de sentido. Em síntese, basicamente, foram percorridos os seguintes passos analíticos: (a) identificação das ideias centrais dos trechos transcritos de todos os artigos; (b) classificação dos sentidos subjacentes às ideias em temas que resumem a produção do conhecimento acerca do assunto estudado; e (c) elaboração de síntese interpretativa dos resultados extraídos do acervo analisado. Nessa síntese interpretativa, foram utilizados como referência teórico-analítica os conceitos de habitus e violência simbólica mencionados na introdução deste trabalho.

\section{Resultados}

\section{Caracterização das fontes}

Foram identificados, 21 artigos, no período de 2006 e 2014, e 15, entre 2015 e 2018 . Em relação ao idioma, 23 artigos eram na língua inglesa, dez em português e três em espanhol. A metade dos estudos foi publicada na Medline e PubMed, e os demais na Lilacs, SciELO e Bdenf. Os artigos selecionados foram provenientes dos Estados Unidos (nove), Brasil (nove), África (cinco), Argentina (dois), Chile (um), Reino Unido (dois), Canadá (dois), Portugal (dois), Alemanha (um), Noruega (um), Suécia (um) e Nova Zelândia (um).

Os 36 artigos que compõem a revisão foram publicados em 32 revistas, sendo 11 brasileiras, 13 estadunidenses, quatro inglesas, uma canadense, uma colombiana, uma sueca e uma norueguesa.

Ao analisar a formação dos primeiros autores dos artigos, essa variou, principalmente, entre: Medicina (20), Enfermagem (três), Psicologia (quatro), Sociologia (seis). Investigou-se ainda a quantidade de autores envolvidos nas publicações, os quais a média era de três autores por artigo (13), seguidas com dois (oito), um autor (cinco), cinco e quatro (três cada um), sete (dois) e seis e nove autores (um cada). Nove artigos mostram uma grande interação entre diferentes áreas de conhecimento e diálogos interdisciplinares.

Em relação à metodologia, dos 36 artigos, 24 se baseiam na abordagem qualitativa, 12 no método quantitativo. Há predomínio da abordagem qualitativa, o que permite inferir que essa metodologia seja mais apropriada para reflexão 
e compreensão de questões subjetivas, e por ser uma metodologia que aborda um nível de realidade que não pode ou não deveria ser quantifica$\mathrm{da}$, pois trabalha o universo dos significados, dos motivos, das aspirações, das crenças, dos valores e das atitudes ${ }^{16}$. Nas publicações quantitativas predominam os estudos comparativos e transversais sobre a população LGBT e as mulheres lésbicas.

Quanto aos participantes do estudo, as pesquisas incluem experiências de mulheres lésbicas, bissexuais, a população LGBT e profissionais de saúde. Se fossem escolhidos somente os estudos que se limitavam a mulheres lésbicas, não haveria um número adequado de artigos para analisar, por isso foram aceitos artigos que também tratam da temática lésbica e bissexuais e LGBT, desde que contemplassem o objetivo da pesquisa.

Com relação ao acervo selecionado, a maioria dos estudos utilizou a amostragem por conveniência, utilizando a técnica de Bola de Neve, para o recrutamento dos participantes.

No conjunto dos artigos, 16 tratam de lésbicas e bissexuais, 09 da população LGBT e 11 de mulheres lésbicas.

No sentido de sintetizar a caracterização das fontes analisadas, foi realizada a construção de um quadro sinóptico (Quadro 1).

\section{Temáticas do acervo}

No conjunto dos artigos analisados, afiguram-se as duas temáticas: (a) Barreiras e dificuldades do acesso de lésbicas à atenção à saúde e (b) Experiências das lésbicas nos serviços de saúde. Cabe observar que - por conta da natureza deste estudo - a análise não se deteve na comparação de perfis epidemiológicos de doenças relacionadas a mulheres homossexuais, bissexuais e heterossexuais, presentes em alguns estudos.

\section{Barreiras e dificuldades do acesso de lésbicas à atenção à saúde}

Dois núcleos de sentidos destacam-se nesta temática: questões relacionadas à revelação de ser lésbica e dificuldades de os serviços e profissionais de saúde lidarem com essas mulheres. Esses sentidos encontram-se imbricados por conta da naturalização da heterossexualidade apontada por algumas das fontes revisadas.

No cenário dessa naturalização, a decisão de revelar sua orientação sexual ("sair do armário”) aparece como uma das principais barreiras para as lésbicas procurarem os serviços de saúde. A lógica no atendimento à saúde de mulheres em geral dos serviços de saúde pauta-se no modelo da heterossexualidade, não levando em conta implícita ou explicitamente - as pessoas consideradas desviantes desse modelo hegemônico, incluindo-se nessa categoria as lésbicas ${ }^{35}$ e isso traz consequências de atitudes discriminatórias que fazer com que lésbicas não acessem os serviços de saúde por se sentirem vulneráveis ${ }^{50}$.

Nesse sentido, a decisão de procurar os serviços de saúde, revelando sua orientação sexual, comumente pode se relacionar a diversos problemas, tais como: tensão e ansiedade ${ }^{52}$; medo da discriminação, do preconceito e da estigmatização ${ }^{19,21,37,45,48,49}$; falta de garantias sobre a confidencialidade ${ }^{31}$; vergonha de exposição do corpo nu para um estranho e exposição de uma intimidade que pode ser desvalorizada socialmente ${ }^{22}$.

Por outro lado, algumas mulheres lésbicas acreditam que a divulgação de sua orientação sexual ao seu médico não afeta negativamente a atenção à sua saúde ${ }^{28}$, podendo criar uma relação de confiança ${ }^{20}$. Mas não garantem que elas recebam um atendimento diferente das heterossexuais, contrariando a suposição de que "sair do armário" seria a solução para melhora dos cuidados à sua saúde ${ }^{22}$. Porém a inclusão da orientação sexual em sistemas de saúde pública, pode contribuir para o reconhecimento das desigualdades de saúde e seus mecanismos ${ }^{25}$.

As dificuldades para lésbicas acessarem os serviços de saúde também se relacionam ao fato de os profissionais nem sempre conseguirem lidar com essas mulheres. Um dos estudos observa que há profissionais de saúde que levam para a sua vida pessoal crenças religiosas que veem a heterossexualidade algo que agrada a Deus ${ }^{46}$. Outras fontes analisadas apontam para a fragilidade da formação dos profissionais que não lhes possibilitam ou lhes causam desconforto para lidar com a diversidade das orientações sexuais ${ }^{40,50,51}$, tornando imperceptível as demandas e as especificidades dessas mulheres. Há outros estudos ainda que tributam à heterossexualidade naturalizada que se encontra - explícita ou implícita na prática assistencial ${ }^{26}$ em geral e na atitude dos profissionais $^{35,52}$.

A partir desse cenário, observam-se inúmeros aspectos que comprometem tanto o acesso quanto a qualidade do atendimento: não abordagem sobre sexualidade em geral com as lésbicas $^{38}$; “apagamento" da orientação sexual homossexual $^{32,47}$; não-debate sobre as doenças sexuais transmissíveis ${ }^{29,42,44}$; ausência de protocolos espe$\operatorname{cíficos}^{50}$; indeterminação sobre necessidades de saúde de lésbicas ${ }^{32}$ e realização de cuidados inseguros, fragmentados e não integral ${ }^{43}$. 
Quadro 1. Distribuição dos Artigos por ano, país, método e foco central.

\begin{tabular}{|c|c|c|c|c|}
\hline Autores dos artigos & Ano & País & Método & Foco central \\
\hline Kerker et al ${ }^{17}$ & 2006 & EUA & Estudo comparativo & Comportamento sexual e acesso à saúde \\
\hline Araújo et $\mathrm{al}^{18}$ & 2006 & Brasil & Estudo de caso & $\begin{array}{l}\text { Experiências lésbicas no atendimento de } \\
\text { saúde }\end{array}$ \\
\hline Heck et $\mathrm{al}^{19}$ & 2006 & EUA & Estudo comparativo & $\begin{array}{l}\text { Cuidados de saúde de lésbicas e mulheres } \\
\text { heterossexuais }\end{array}$ \\
\hline Mravcak $^{20}$ & 2006 & EUA & Estudo documental & $\begin{array}{l}\text { Acesso de lésbicas e mulheres bissexuais à } \\
\text { saúde }\end{array}$ \\
\hline Tjepkema $^{21}$ & 2008 & Canadá & Estudo comparativo & $\begin{array}{l}\text { Cuidados de saúde de gays, lésbicas e } \\
\text { bissexuais }\end{array}$ \\
\hline Barbosa e Facchini ${ }^{22}$ & 2009 & Brasil & Estudo etnográfico & Cuidados de saúde entre lésbicas \\
\hline Matebeni et $\mathrm{al}^{23}$ & 2009 & África & Estudo exploratório & HIV/AIDS e experiências lésbicas \\
\hline $\begin{array}{l}\text { Bjorkman e } \\
\text { Malterud }^{24}\end{array}$ & 2009 & Noruega & Estudo exploratório & $\begin{array}{l}\text { Orientação sexual e acesso aos serviços de } \\
\text { saúde }\end{array}$ \\
\hline Dilley et $\mathrm{al}^{25}$ & 2010 & EUA & Estudo comparativo & Acesso de gays, lésbicas e bissexuais \\
\hline Fish e Bewleys ${ }^{26}$ & 2010 & $\begin{array}{l}\text { Reino } \\
\text { Unido }\end{array}$ & $\begin{array}{l}\text { Estudo exploratório- } \\
\text { descritivo qualitativo }\end{array}$ & $\begin{array}{l}\text { Direitos humanos para saúde de lésbicas e } \\
\text { bissexuais }\end{array}$ \\
\hline Austin e Irwin ${ }^{27}$ & 2010 & EUA & Estudo comparativo & $\begin{array}{l}\text { Utilização de serviços de saúde entre lésbicas e } \\
\text { mulheres em geral }\end{array}$ \\
\hline Formby $^{28}$ & 2011 & $\begin{array}{l}\text { Reino } \\
\text { Unido }\end{array}$ & $\begin{array}{l}\text { Estudo exploratório- } \\
\text { descritivo qualitativo }\end{array}$ & Saúde sexual de lésbicas e mulheres bissexuais \\
\hline Charlton et $\mathrm{al}^{29}$ & 2011 & EUA & Estudo comparativo & Orientação sexual e saúde reprodutiva. \\
\hline Marques et $\mathrm{al}^{30}$ & 2012 & Brasil & Estudo reflexivo & Relação lésbicas-serviço de saúde \\
\hline Corbett et $\mathrm{al}^{31}$ & 2013 & Canadá & $\begin{array}{l}\text { Estudo exploratório- } \\
\text { descritivo qualitativo }\end{array}$ & Lésbicas e serviços de fertilidade \\
\hline Carvalho et $\mathrm{al}^{32}$ & 2013 & Brasil & Estudo documental & Políticas de saúde para lésbicas \\
\hline Carvalho et $\mathrm{al}^{33}$ & 2013 & Brasil & $\begin{array}{l}\text { Estudo exploratório- } \\
\text { descritivo qualitativo }\end{array}$ & Experiências de lésbicas e serviços de saúde. \\
\hline Mosack et $\mathrm{al}^{34}$ & 2013 & EUA & Estudo comparativo & $\begin{array}{l}\text { Cuidados de saúde de lésbicas e mulheres } \\
\text { heterossexuais }\end{array}$ \\
\hline Araújo e Penna ${ }^{35}$ & 2014 & Brasil & Estudo reflexivo & $\begin{array}{l}\text { Identidade sexual e de gênero no cotidiano do } \\
\text { serviço de saúde }\end{array}$ \\
\hline Brown et $\mathrm{al}^{36}$ & 2014 & Argentina & $\begin{array}{l}\text { Estudo exploratório- } \\
\text { descritivo qualitativo }\end{array}$ & $\begin{array}{l}\text { Experiências de saúde de lésbicas e serviços } \\
\text { de saúde }\end{array}$ \\
\hline Marques et al ${ }^{37}$ & 2014 & Portugal & Estudo exploratório & $\begin{array}{l}\text { Experiências de lésbicas em atendimentos } \\
\text { médicos. }\end{array}$ \\
\hline Poteat et $\mathrm{al}^{38}$ & 2015 & África & Estudo transversal & $\begin{array}{l}\text { Experiências de lésbicas, mulheres bissexuais e } \\
\text { heterossexuais nos serviços de saúde }\end{array}$ \\
\hline $\operatorname{Rubin}^{39}$ & 2015 & EUA & Estudo exploratório & $\begin{array}{l}\text { Desigualdades de saúde entre pacientes gays, } \\
\text { lésbicas e bisssexuais }\end{array}$ \\
\hline Hirsch et $\mathrm{al}^{40}$ & 2016 & Alemanha & $\begin{array}{l}\text { Estudo exploratório- } \\
\text { descritivo } \\
\text { quantitativo } \\
\end{array}$ & Saúde de lésbicas e atenção primária \\
\hline Videla e Munoz ${ }^{41}$ & 2016 & Chile & $\begin{array}{l}\text { Estudo transversal } \\
\text { exploratório }\end{array}$ & Vivências de lésbicas na atenção perinatal \\
\hline Daly et $\mathrm{al}^{42}$ & 2016 & África & Estudo documental & $\begin{array}{l}\text { Violação sexual, } \\
\text { Lésbicas e bissexuais e direitos à saúde }\end{array}$ \\
\hline Munson e Cook ${ }^{43}$ & 2016 & $\begin{array}{l}\text { Nova } \\
\text { Zelândia }\end{array}$ & Estudo descritivo & Lésbicas e bissexuais no acesso a saúde \\
\hline Silberman et a ${ }^{44}$ & 2016 & Argentina & $\begin{array}{l}\text { Estudo descritivo de } \\
\text { corte transversal }\end{array}$ & $\begin{array}{l}\text { Dificuldades de acesso à saúde sexual de } \\
\text { lésbicas }\end{array}$ \\
\hline Bränström et $\mathrm{al}^{45}$ & 2016 & EUA & Estudo comparativo & $\begin{array}{l}\text { Desigualdades de saúde entre gays, lésbicas e } \\
\text { bissexuais }\end{array}$ \\
\hline
\end{tabular}


Quadro 1. Distribuição dos Artigos por ano, país, método e foco central.

\begin{tabular}{|l|c|l|l|l|}
\hline Autores dos artigos & Ano & \multicolumn{1}{|c|}{ País } & \multicolumn{1}{|c|}{ Método } & \multicolumn{1}{|c|}{ Foco central } \\
\hline Vitiritti et al $^{46}$ & 2016 & Brasil & $\begin{array}{l}\text { Estudo exploratório- } \\
\text { descritivo qualitativo }\end{array}$ & $\begin{array}{l}\text { Discursos de profissionais de saúde acerca da } \\
\text { diversidade sexual }\end{array}$ \\
\hline Moscheta et al ${ }^{47}$ & 2016 & Brasil & $\begin{array}{l}\text { Estudo exploratório- } \\
\text { descritivo qualitativo }\end{array}$ & $\begin{array}{l}\text { Relação entre profissional- usuário gays/ } \\
\text { lésbicas/bissexuais }\end{array}$ \\
\hline${\text { Alpert et al }{ }^{48}}^{48}$ & 2017 & EUA & Estudo exploratório & GLBT e acesso a saúde \\
\hline Müller $^{49}$ & 2017 & África & $\begin{array}{l}\text { Estudo exploratório- } \\
\text { descritivo qualitativo }\end{array}$ & $\begin{array}{l}\text { Cuidados de saúde para gays, lésbicas, } \\
\text { bissexuais e transsexuais }\end{array}$ \\
\hline Müller $^{50}$ & 2017 & África & $\begin{array}{l}\text { Estudo exploratório- } \\
\text { descritivo qualitativo }\end{array}$ & GLBT e acesso aos serviços de saúde \\
\hline Rufino et al ${ }^{51}$ & 2018 & Brasil & $\begin{array}{l}\text { Estudo exploratório- } \\
\text { descritivo qualitativo }\end{array}$ & $\begin{array}{l}\text { Experiências lésbicas durante atendimento } \\
\text { ginecológico }\end{array}$ \\
\hline Rufino et al ${ }^{52}$ & 2018 & Brasil & Estudo transversal & $\begin{array}{l}\text { Práticas sexuais e Cuidados de saúde de } \\
\text { lésbicas }\end{array}$ \\
\hline
\end{tabular}

Fonte: Elaborado pelos autores.

\section{Experiências das lésbicas nos serviços de saúde}

Com base na literatura analisada, três núcleos de sentidos podem sintetizar a experiência das lésbicas quando conseguem ser atendidas nos serviços de saúde: atendimento desigual, invisibilidade e constrangimento.

Quando acessam os serviços de saúde, em comparação com as mulheres heterossexuais, para alguns estudos analisados, as lésbicas: têm um atendimento mais fragilizado ${ }^{17}$; mostram menores taxas de realização de teste de Papanicolau $^{27,33}$; são discriminadas na atenção perinatal quando buscam constituir famílias homoparentais ${ }^{41}$ e recebem menos orientações ou esclarecimentos sobre suas dúvidas ${ }^{27,33}$.

A literatura consultada também associa a invisibilidade à experiência de lésbicas durante seu atendimento nos serviços de saúde. Tal associação baseia-se em algumas situações, tais como: silenciamento sobre a sua sexualidade ${ }^{23}$; ausência de acolhimento que permita uma escuta de suas vivências ${ }^{34,36} \mathrm{e}$ formação de vínculos efetivo ${ }^{39}$; formas verbais e publicadas na área de educação em saúde baseadas em princípios heterossexuais ${ }^{31,35}$ e não-estabelecimento de relações colaborativas e de confiança ${ }^{40}$.

Essa invisibilidade - promovida de forma consciente ou inconsciente - pode comprometer a experiência das lésbicas durante o atendimento por parte dos serviços de saúde das lésbicas. Um dos estudos analisados considera que, no sistema de saúde privado, em que se paga pelo atendimento recebido, há maior visibilidade da homossexualidade ${ }^{41}$.
Ainda em termos de experiências de lésbicas relacionadas aos serviços de saúde, dois estudos relatam situações que podem ser vistas como constrangedoras para essas mulheres. Um deles registrou experiências negativas de lésbicas no passado com os profissionais durantes os cuidados de saúde ${ }^{21}$. Outro constatou que as informantes relataram sentirem-se forçadas a revelar sua orientação sexual quando era questionada em relação a ter vida sexualmente ativa e não usar contraceptivos ${ }^{24}$.

\section{Síntese interpretativa}

Em geral, os estudos analisados destacam a necessidade do reconhecimento da existência das demandas e especificidades da saúde das mulheres lésbicas para que essas mulheres possam ter condições de acessar os serviços de saúde e terem um atendimento de qualidade. No campo das políticas, em alguns países, observam-se avanços que, de certa forma, norteiam esse reconhecimento. No Brasil, por exemplo, destacamse documentos, tais como Política Nacional de Saúde Integral de LGBTT ${ }^{5}$, Dossiê de Saúde das Mulheres Lésbicas ${ }^{3}$, Relatório da Oficina de Atenção à Saúde de Mulheres Lésbicas ${ }^{53}$ e Bissexuais, Cartilha Mulheres Lésbicas e Bissexuais: direitos, saúde e participação social ${ }^{54}$ e Cartilha Se você é lésbica a/o profissional de saúde precisa saber ${ }^{55}$.

Em contrapartida, em geral, os estudos apontam, implícita ou explicitamente, que o habitus heteronormativo, presente nas práticas de saúde, realizam a produção dos cuidados voltada para as lésbicas como se elas fossem heterossexuais. 
Isso tanto pode levar à ideia da naturalização da heterossexualidade que pensa a existência lésbica como desviante, quanto o não reconhecimento de vivências sexuais e de gênero plurais, como as das lésbicas.

Junto a isso, as fontes estudas também destacam que, além dos diversos preconceitos que as mulheres lésbicas enfrentam nos espaços públicos e privados e que geram violências físicas e psicológicas, a violência simbólica também se encontra presente nos serviços de saúde, uma vez que é comum, esses serviços não considerarem as demandas e as especificidades dessas mulheres, contribuindo para o apagamento de sua existência.

Mudanças nesse cenário implicam em ações sociais que extrapolem a área da saúde e que abalem as instituições para que se possa enfrentar o habitus que desconsidera lésbicas e outras orientações que contestam a hegemonia hetonormativa. Dentre essas ações, destacam-se as relacionadas aos movimentos sociais, principalmente ao movimento gay e lésbico. Segundo Bourdieu ${ }^{11}$ a ação desse movimento não se deve limitar a rupturas simbólicas, ainda que algumas delas sejam eficazes como bem ele observa. Para esse pensador, o movimento gay e lésbico "tem que operar e impor transformações das categorias incorporadas (dos esquemas de pensamento) que, através da educação, conferem um estatuto de realidade evidente [...] às categorias sociais que elas produzem”. Mas, ele adverte que a obtenção do reconhecimento da particularidade (passando da invisibilidade para a visibilidade) pode implicada sua anulação como movimento. Nesse sentido, assim como no caso de outros movimentos nascidos dos grupos dominados e estigmatizados, o movimento em questão vive uma contradição estrutural pendular entre "a invisibilidade e a exibição, entre a anulação e a celebração de diferença que faz com que [...] adotem, segundo as circunstâncias, uma ou outra estratégia..."11.

\section{Considerações finais}

Os artigos contidos na revisão apontam que as desigualdades de acesso e as barreiras na atenção ao se revelar lésbica, tem relação direta com a normativas de gênero e que esta influencia diretamente na revelação ou não de sua sexualidade. Apesar dos avanços em relação a políticas e protocolos de atendimento a essa população, para a garantia da equidade e de integralidade, as diversidades sexual e de gênero devem ser amplamente debatidas nos espaços sociais, de formação e de atenção à saúde.

A construção de políticas públicas de saúde, criação de protocolos e intervenções a partir de um olhar heteronormativo dificulta a construção de vínculos e o cuidado, produzindo um atendimento fragmentado que contribui para exclusão e violência simbólica.

Conforme o referencial teórico de Bourdieu, uma possibilidade de modificar esse habitus, está na intensificação da abordagem da temática nos espaços de diálogo entre o movimento lésbico, o governo e a academia que fortaleça a criação de políticas, protocolos e estudos sobre a saúde das lésbicas. E a inserção nos cursos de formação técnica, de graduação e reforçada com a ampliação nas residências e na construção de especializações voltadas para o acolhimento e conhecimento das demandas e especificidades das mulheres lésbicas.

Os profissionais de saúde no desenvolvimento de habilidades e competências, deve conhecer e valorizar as necessidades reais da população que está sob seus cuidados, através a criação de um ambiente acolhedor, que favoreça a construção de vínculos e uma relação profissional usuária aberta, em um espaço marcado de estigmas, discriminação e medos, que constitui o rompimento com a violência institucionalizada quando essas mulheres procuram os serviços de saúde, que respeite à singularidade desconstrua todas as formas de violência contra as lésbicas e que gera exclusão e de discriminação, constituindo um cuidado inclusivo, integral e humanizado. 


\section{Colaboradores}

AN Silva trabalhou na concepção, pesquisa, metodologia e redação final. $\mathrm{R}$ Gomes orientador da doutoranda participou de todas as fases do artigo.

\section{Referências}

1. Assis MMA, Jesus WLA. Acesso aos serviços de saúde: abordagens, conceitos, políticas e modelo de análise. Cien Saude Colet 2012; 17(11):2865-2875.

2. Portella AP. Direitos Sexuais e Necessidades de Saúde de Lésbicas e Mulheres Bissexuais na Percepção de Profissionais de Saúde e Usuárias do SUS. In: Brasil. Ministério da Saúde (MS). Atenção integral à saúde de mulheres lésbicas e bissexuais. Brasília: MS; 2014. p. 23-30.

3. Facchini R, Barbosa RM. Dossiê saúde das mulheres lésbicas: promoção da equidade e da integralidade. Belo Horizonte: Rede Saúde; 2006.

4. Valadão RC, Gomes R. A homossexualidade feminina no campo da saúde: da invisibilidade à violência. $P h y$ sis 2011; 21(4):1451-1467.

5. Brasil. Ministério da Saúde (MS). Política Nacional de Saúde Integral de Lésbicas, Gays, Bissexuais, Travestis e Transexuais. Brasília: MS; 2013.

6. Bourdieu P. A Economia das Trocas Simbólicas. São Paulo: Perspectiva; 1992.

7. Bourdieu P. O Poder Simbólico. Rio de Janeiro: Editora Bertrand Russel; 1998.

8. Bourdieu P. Meditações pascalianas. Rio de Janeiro: Bertrand Brasil; 2001.

9. Bourdieu P. Esboço de uma teoria da prática. Oiras [Portugal]: Celta Editora, 2002.

10. Bourdieu P. O senso prático. Petrópolis: Vozes; 2009.

11. Bourdieu P. A dominação masculina. Rio de Janeiro: Bertrand Brasil;1999.

12. Lima TCS, Mioto RCT. Procedimentos metodológicos na construção do conhecimento científico: a pesquisa bibliográfica. Rev Katálysis 2007; 10(n. esp.):37-45.

13. Brasil. Ministério da saúde (MS). Secretaria de Atenção à Saúde. Departamento de ações Programáticas estratégicas. Política nacional de atenção integral à saúde da mulher: princípios e diretrizes. Brasília: MS; 2004.

14. Bardin L. Análise de conteúdo. Lisboa: Edições 70; 1979.

15. Gomes R. A análise de dados em pesquisa qualitativa. In: Minayo MCS, organizadora. Pesquisa social: teoria, método e criatividade. Petrópolis: Editora Vozes; 2002. p. 67-80.

16. Minayo MCS. Ciência, técnica e arte: o desafio da pesquisa social. In: Minayo MCS, organizadora. Pesquisa social: teoria, método e criatividade. Petrópolis: Editora Vozes; 2002. p. 21-22.

17. Kerker BD, Mostashari F, Thorpe L. Health care access and utilization among women who have sex with women: sexual behavior and identity. J Urban Health 2006; 83(5):970-979.

18. Araújo MAL, Galvão MTG, Saraiva MMM, Albuquerque AD. Relação usuária-profissional de saúde: experiência de uma mulher homossexual em uma unidade de saúde de referência de Fortaleza. Esc Anna Nery 2006; 10(2):323-327.

19. Heck JE, Sell RL, Gorin SS. Health care access among individuals involved in same-sex relationships. Am J Public Health 2006; 96(6):1111-1118.

20. Mravcak SA. Primary care for lesbians and bisexual women. Am Fam Physician 2006; 74(2):279-286.

21. Tjepkema M. Health care use among gay, lesbian and bisexual Canadians. Health Rep 2008; 19(1):53-64. 
22. Barbosa RM, Facchini R. Acesso a cuidados relativos à saúde sexual entre mulheres que fazem sexo com mulheres em São Paulo, Brasil. Cad Saude Publica 2009; 25(Supl. 2):s291-s300.

23. Matebeni Z, Camargo TMCR, Camargo Júnior KR, Dias LF. All sexed up: a resposta de mulheres lésbicas negras jovens ao sexo (mais) seguro em Johannesburg, África do Sul. Physis 2009; 19(2):333-348.

24. Bjorkman M, Malterud K. Lesbian women's experiences with health care: a qualitative study. Scand J Prim Health Care 2009; 27(4):238-243.

25. Dilley JA, Simmons KW, Boysun MJ, Pizacani BA, Stark MJ. Demonstrating the importance and feasibility of including sexual orientation in public health surveys: health disparities in the Pacific Northwest. Am J Public Health 2010; 100(3):460-467.

26. Fish J, Bewley S. Using human rights-based approaches to conceptualise lesbian and bisexual women's health inequalities. Health Soc Care Community 2010; 18(4):355-362.

27. Austin EL, Irwin JA. Health behaviors and health care utilization of southern lesbians. Womens Health Issues 2010; 20(3):178-184.

28. Formby E. Lesbian and bisexual women's human rights, sexual rights and sexual citizenship: negotiating sexual health in England. Cult Health Sex 2011; 13(10):1165-1179.

29. Charlton BM, Corliss HL, Missmer SA, Frazier AL, Rosario M, Kahn JA, Austin SB. Reproductive Health Screening Disparities and Sexual Orientation in a Cohort Study of U.S. Adolescent and Young Adult Females. J Adolesc Health 2011; 49(5):505-510.

30. Marques AM, Oliveira JM, Nogueira C. A população lésbica em estudos da saúde: contributos para uma reflexão crítica. Cien Saude Colet 2013; 18(7):20372047.

31. Corbett SL, Frecker HM, Shapiro HM, Yudin MH. Access to fertility services for lesbian women in Canada. Fertil Steril 2013; 100(4):1077-1080.

32. Carvalho CS, Calderaro F, Souza SJ. O dispositivo "saúde de mulheres lésbicas": (in)visibilidade e direitos. Rev Psicol Polit 2013; 13(26):111-127.

33. Nóbrega BSM, Oliveira JL, Almeida RO, Abdalla FTM, Nichiata LYI, Carvalho PMG. Prevention of sexually transmitted diseases by homosexual and bisexual women: a descriptive study. Online Braz J Nurs 2013; 12(4):931-941.

34. Mosack KE, Brouwer AM, Petroll AE. Sexual identity, identity disclosure, and health care experiences: is there evidence for differential homophobia in primary care practice? Womens Health Issues 2013; 23(6):e341-e346.

35. Araújo LM, Penna LHG. A relação entre sexo, identidades sexual e de gênero no campo da saúde da mulher. Rev Enferm UERJ 2014; 22(1):134-138.

36. Brown JL, Pecheny M, Tamburrino MC, Conde LL, Perrotta GV, Capriati A, Andia AM, Mario S, Ibarlucia I. Atención ginecológica de lesbianas y bisexuales: notas sobre el estado de situación en Argentina. Atención ginecológica de lesbianas y bisexuales: notas sobre el estado de situación en Argentina. Interface (Botucatu) $2014 ; 18(1): 673-684$
37. Marques AM, Nogueira C, Oliveira JM. Lesbians on medical encounters: tales of heteronormativity, deception, and expectations. Health Care Women Int 2015; 36(9):988-1006.

38. Poteat TC, Logie CH, Adams D, Mothopeng T, Lebona J, Letsie P, Baral S. Stigma, sexual health, and human rights among women who have sex with women in Lesotho. Reprod Health Matters 2015; 23(46):107-116

39. Rubin R. Minimizing Health Disparities Among LGBT Patients. JAMA 2015; 313(1):15-17.

40. Hirsch O, Löltgen K, Becker A. Lesbian womens' access to healthcare, experiences with and expectations towards GPs in German primary care. BMC Fam Pract 2016; 17(1):162.

41. Videla CF, Muñoz AV. Experiences of lesbian users about the process of perinatal care in the metropoli$\tan$ region of Chile in 2016. Interface (Botucatu) 2018; 22(66):777-778.

42. Daly F, Neil S, Samantha W. Sexual rights but not the right to health? Lesbian and bisexual women in South Africa's National Strategic Plans on HIV and STIs. Reprod Health Matters 2016; 24(47):185-194.

43. Munson S, Cook C. Lesbian and bisexual women's sexual healthcare experiences. J Clin Nurs 2016; 25(2324):3497-3510.

44. Silberman P, Buedo PE, Burgos LM. Barreras en la atención de la salud sexual en Argentina: percepción de las mujeres que tienen sexo con mujeres. Rev Salud Publica 2016; 18(1):1-12.

45. Bränström R, Hatzenbuehler ML, Pachankis JE. Sexual orientation disparities in physical health age and gender effects in a population-based study. Soc Psychiatry Psychiatr Epidemiol 2016; 51(2):289-301.

46. Vitiritti B, Andrade SMO, Peres JEC. Diversidade sexual e relações profissionais: concepções de médicos e enfermeiros. Temas Psicol 2016; 24(4):1389-1405.

47. Moscheta MS, Febole DS, Anzolin B. Visibilidade seletiva: a influência da heterossexualidade compulsória nos cuidados em saúde de homens gays e mulheres lésbicas e bissexuais. Saude Transf 2016; 7(3):71-83.

48. Alpert AB, CichoskiKelly EE, Fox AD. What lesbian, gay, bisexual, transgender, queer, and intersex patients say doctors should know and do: a qualitative study. $J$ Homosex 2017; 64(3):1368-1389.

49. Müller A. Scrambling for access: availability, accessibility, acceptability and quality of healthcare for lesbian, gay, bisexual and transgender people in South Africa. BMC Int Health Hum Rights 2017; 17(1):16.

50. Müller A. Health for all? sexual orientation, gender identity, and the implementation of the right to access to health care in south Africa. Health Hum Rights 2016; 18(2):195-208

51. Rufino AC, Madeiro A, Trinidad AS, Rodrigues dos Santos R, Freitas I. Disclosure of sexual orientation among women who have sex with women during gynecological care: a qualitative study in Brazil. J Sex Med 2018; 15(7):966-973.

52. A C, Madeiro A, Trinidad A, Santos R, Freitas I. Práticas sexuais e cuidados em saúde de mulheres que fazem sexo com mulheres: 2013-2014. Epidemiol Serv Saude 2018; 27(4):e2017499 
53. Brasil. Ministério da Saúde (MS). Secretaria de Políticas para as Mulheres. Atenção Integral à Saúde de Mulheres Lésbicas e Bissexuais. Relatório da Oficina Atenção à Saúde de Mulheres Lésbicas e Bissexuais: Brasília: MS; 2014.

54. Brasil. Ministério da Saúde (MS). Secretaria de Gestão Estratégica e Participativa. Departamento de Apoio à Gestão Participativa. Mulheres lésbicas e bissexuais: direitos, saúde e participação social. Brasília: MS; 2013.

55. Lemos AM, Santos MA, Barbosa R, organizadoras. Cartilha Se você é lésbica a/o profissional de saúde precisa saber. Pernambuco: COMLESBI; 2016.

Artigo apresentado em 17/09/2019

Aprovado em 04/12/2019

Versão final apresentada em 06/12/2019

Editores-chefes: Maria Cecília de Souza Minayo, Antônio Augusto Moura da Silva 